\title{
Uso de Mesa Tangível na Educação Inclusiva
}

\author{
Evandro Preuss ${ }^{1}$, Martha Barcellos Vieira ${ }^{1}$, Katia Soares Coutinho', \\ Renato Ventura Bayan Henriques ${ }^{1}$, Sandra Baldassarri ${ }^{2}$ \\ ${ }^{1}$ Programa de Pós-Graduação em Informática na Educação \\ Universidade Federal do Rio Grande do Sul (UFRGS) - Porto Alegre - RS - Brazil \\ ${ }^{2}$ Departamento de Informática e Ingeniería de Sistemas \\ Universidad de Zaragoza - Zaragoza - Spain \\ \{evandro.preuss, barcellosvieira.martha, katsoarescoutinho\}@gmail.com, \\ renatobayan@gmail.com, sandra@unizar.es
}

\begin{abstract}
Tangible tabletops can be used for educational purposes with considerable advantages, especially when it involves children with intellectual disabilities or autism. The main problems for its use in the educational environment are the lack of equipment and software and the difficulty of creating tangible resources, mainly by teachers who do not master the logic of computer programming. This paper presents a proposal for a low-cost tangible tabletop use in inclusive education, involving a specification to build a tangible tabletop, a software for the creation of activities by the teachers themselves and aspects related to teacher training to use this technology.
\end{abstract}

Resumo. Mesas tangíveis podem ser utilizadas para fins educacionais com consideráveis vantagens, especialmente quando envolve crianças com deficiência intelectual ou autismo. Os principais problemas para sua utilização no ambiente educacional são a falta de equipamentos e softwares e a dificuldade de criação de recursos tangiveis, principalmente por professores que não dominam a lógica da programação. Este trabalho apresenta uma proposta de uso de mesa tangível de baixo custo na educação inclusiva, envolvendo uma especificação para construção da mesa tangivel, um software para criação das atividades pelos próprios professores e os aspectos relacionados à formação de professores para a utilização desta tecnologia.

\section{Introdução}

A utilização de recursos tecnológicos em ambientes de ensino-aprendizagem pode proporcionar uma dinâmica de interação através de um ambiente mais rico e atrativo para a mediação entre sujeitos e destes com os objetos de aprendizagem. A utilização desses recursos torna-se ainda mais relevante e potencializa-se quando envolve o processo de ensino-aprendizagem de crianças com deficiência intelectual ou autismo.

Os avanços nas pesquisas de ambientes computacionais para apoio e suporte ao ensino, especialmente para crianças com algum tipo de deficiência, envolvem o uso e o aprimoramento de interfaces tangíveis a partir da manipulação de objetos reais para interação com o ambiente computacional. $O$ uso de interfaces tangíveis para fins educacionais apresenta consideráveis vantagens e pode proporcionar um incremento no 
engajamento e na motivação dos alunos, além de se revelarem recursos de tecnologia assistiva particularmente adequados para fins educacionais.

A necessidade legal e moral de garantir o direito universal à educação para toda e qualquer pessoa, com ou sem deficiência, num contexto inclusivo, requer uma transformação nas práticas pedagógicas de modo a garantir o acesso, a permanência e a aprendizagem de todos. Essa inclusão traz novos desafios para a escola e para os professores, que precisam estar preparados e dispor de técnicas, metodologias e recursos para proporcionar uma educação ampla e completa para esses alunos. Para que a inclusão se concretize, há a necessidade de uso de tecnologias assistivas, capacitação dos recursos humanos e adequação do espaço escolar e de seus equipamentos e materiais pedagógicos

O problema enfrentado atualmente no uso de mesas tangíveis na educação é que os produtos comerciais disponíveis não são financeiramente viáveis, além de exigirem conhecimento técnico e domínio de lógica de programação. Isso impede que os próprios professores criem seus recursos educacionais para as mesas, uma vez que são eles que conhecem as reais necessidades de cada aluno e quais atividades são mais adequadas para cada caso.

A proposta desta pesquisa está voltada para o uso da tecnologia tangível como recurso e estratégia na formação dos sujeitos participantes, propiciando a compensação social da deficiência (VYGOTSKY, 2012) ao permitir que o sujeito supere barreiras a partir da mediação pedagógica apoiada com tecnologia. A mesa tangível pode ser utilizada como um instrumento de mediação tecnológica, sendo utilizada em atividades educacionais e colaborativas para pessoas com ou sem deficiência, mas pode também ser uma tecnologia assistiva com um potencial maior para crianças com deficiência intelectual ou autismo.

Para usufruir do potencial que as mesas tangíveis proporcionam, este trabalho apresenta uma especificação para a montagem de uma mesa tangível de baixo custo juntamente com a descrição de um editor de aplicações desenvolvido especificamente para esse contexto, podendo ser utilizado de modo intuitivo e interativo por professores, sem necessidade de conhecimentos de lógica de programação. Além disso, aborda questões relacionadas à formação de professores para o uso deste tipo de tecnologia na educação inclusiva.

\section{Mesa Tangível}

A redução nos custos dos dispositivos e o aumento na capacidade de processamento computacional têm proporcionado o desenvolvimento de interfaces interativas tangíveis (TUI - Tangible User Interface) (ULLMER e ISHII, 2000). As TUIs permitem a utilização de objetos físicos reais na interface computacional com os usuários, atuando como controle na manipulação de um objeto ou informação.

Uma mesa tangível é uma interface de computador com uma superfície horizontal, na qual as entradas dos usuários são capturadas através do posicionamento de objetos físicos reais, identificados com marcadores fiduciais em sua base. A saída do sistema é exibida na superfície da mesa por telas de LCD ou projetores multimídia posicionados abaixo ou acima da mesma. Uma mesa tangível pode ser utilizada em 
tarefas cognitivas que envolvem representações visuais, sonoras, táteis e simbólicas de objetos para realizar experimentações, apresentar narrativas e resolver desafios e jogos. Uma vantagem da interação tangível é que ela também permite atividades colaborativas nas quais as pessoas interagem naturalmente na superfície da mesa.

Diversos experimentos com mesas tangíveis foram desenvolvidos e relatam sua utilidade e viabilidade, como o instrumento musical eletrônico ReacTable (JORDÀ et al., 2006), as mesas tangíveis para atividades educacionais NIKVision (MARCO et al., 2013) e o KitVision (BONILLO et al., 2019). O KitVision é uma das poucas propostas que disponibiliza um software para criação de aplicações tangíveis, embora tenha algumas limitações na criação de atividades e seja baseado em tecnologia Flash, suportando apenas vídeos e animações neste formato. Também, se destacam os trabalhos com mesas tangíveis Combinatorix (SCHNEIDER et al., 2012), o Immertable (BALDASSARRI et al., 2017), o ToyVision (MARCO et al., 2016) e o Ambiente Virtual Tangível (GLUZ et al., 2018).

Os ambientes tangíveis expandem as possibilidades de fornecer auxílios nos processos de aprendizagem, pois acoplam representações digitais a objetos físicos, o que é particularmente útil para a formação de conceitos e generalização das crianças com deficiência intelectual ou autismo. Diversos trabalhos exploram esse potencial, como as pesquisas envolvendo crianças com deficiência intelectual e a importância do feedback (FALCÃO, 2018), o sistema de reabilitação cognitiva com uma interface de mesa tangível (JUNG et al., 2013), os jogos interativos para promover a comunicação e a atenção de crianças com TEA (BALDASSARRI et al., 2018), o uso da mesa tangível NIKVision para as atividades (CEREZO et al., 2016) e jogos para crianças com TDAH (CEREZO et al., 2019), o jogo que simula as rotinas diárias para crianças com deficiência cognitiva (KOROZI et al., 2018) e a utilização de jogos de computador para avaliar e auxiliar no diagnóstico clínico e intervenção educacional de crianças com TEA (CHEN et al., 2019).

Existem poucas e caras soluções comerciais de mesas tangíveis. Em 2007 foi lançada a Microsoft Surface, que reconhecia objetos físicos e permitia o controle direto do conteúdo, como fotos, músicas e mapas, fornecendo interação com todas as formas de conteúdo digital através de gestos naturais, toques e objetos físicos. No final de 2011, a Samsung lançou a mesa SUR40, com preço inicial US\$ 8400 nos EUA. A mesa possuía um computador embutido, programação baseada no SDK do Surface 2.0 da Microsoft e uma tela multitoque de 40 polegadas com tecnologia PixelSense, onde cada pixel do LCD emite um feixe de infravermelho que reflete um objeto de volta para o sensor, permitindo que os mesmos sejam detectados (Samsung Electronics, 2011). Apesar de ter recebido premiação pela inovação tecnológica, a mesa tangível SUR40 não foi um sucesso de vendas, seu uso foi restrito e ela foi descontinuada. O alto custo e a exigência de um elevado nível de conhecimento para o desenvolvimento de aplicações podem ter sido alguns dos motivos da sua não utilização em atividades e ambientes educacionais, onde há a necessidade de criação de atividades específicas, de acordo com as necessidades de cada professor e aluno.

Os monitores MultiTaction também são uma solução comercial completa envolvendo hardware e software e podem ser usados como mesa interativa com suporte a toque, multitoque e objetos com marcadores fiduciais. Um monitor MultiTaction 
reconhece e identifica os marcadores usando algoritmos de visão computacional e rastreamento. Todavia, o problema desta solução também é o alto custo e a complexidade para criação de novas aplicações para uso educacional.

Por outro lado, há uma variedade de tecnologias, dispositivos e técnicas usados para prototipar e implementar mesas tangíveis. As três tecnologias de implementação que são frequentemente usadas no desenvolvimento de TUIs são a visão computacional, RFID e microcontroladores (SHAER e HORNECKER, 2009). O trabalho de Schöning et al. (2010) fornece instruções para criar mesas tangíveis, discutindo os prós e contras das diferentes abordagens. Enquanto os sistemas comerciais ainda não se consolidam como uma solução tecnológica e financeiramente viável, os pesquisadores e desenvolvedores ainda precisam moldar e controlar o hardware subjacente.

\section{Métodos}

Como parte de um projeto de pesquisa-ação, nos últimos três anos foram construídos dois protótipos e três mesas tangíveis, baseadas em visão computacional conforme as descrições de Shaer e Hornecker (2009) e adaptadas para a realidade da educação inclusiva, nas quais foram testadas diferentes formas de montagem, câmeras, iluminadores e projetores. Destas mesas, uma está num laboratório de tecnologia assistiva, utilizado por alunos de graduação da área de educação de uma universidade pública e as outras duas (fig. 1) estão na sala de recursos do atendimento educacional especializado de duas escolas públicas.

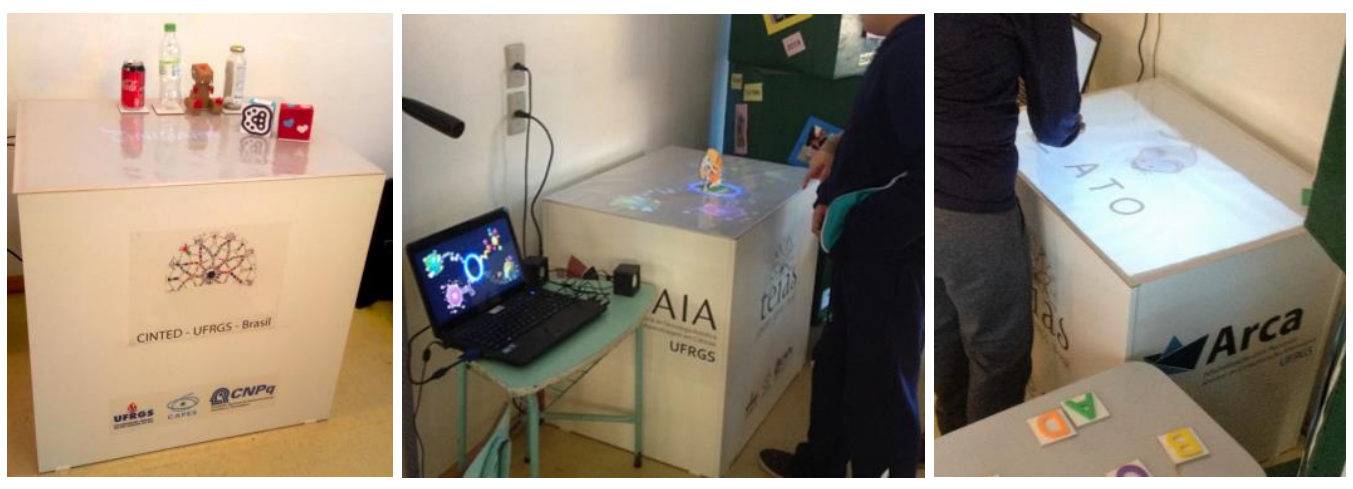

Figura 1. Uso da mesa tangível desenvolvida

Para o funcionamento e uso das mesas tangíveis foi desenvolvido um sistema com um editor de aplicações e um player (PREUSS et al., 2019). O player executa as aplicações e faz o reconhecimento das interações utilizando o rastreamento dos marcadores fiduciais dos objetos colocados sobre a mesa baseado no ReacTIVision (KALTENBRUNNER, 2009). O desenvolvimento do software utilizou métodos ágeis, envolvendo processo incremental, com a criação de protótipos funcionais do sistema a cada 2 ou 3 semanas. A especificação de requisitos foi realizada durante a utilização do editor do KitVision (BONILLO et al., 2019) por dois meses, com 25 alunos de duas turmas de uma disciplina de educação inclusiva, numa universidade pública. Durante o processo de desenvolvimento, nos últimos dois anos, a cada nova versão, o sistema e o editor foram utilizados pelos alunos das turmas subsequentes da mesma disciplina, para que as funcionalidades implementadas fossem testadas e aprimoradas. A usabilidade do 
editor e a autonomia e motivação para uso do sistema foram avaliados através dos questionários SUS (System Usability Scale) e IMI (Intrinsic Motivation Inventory).

As três mesas estão sendo utilizadas na pesquisa e nas atividades educacionais. No laboratório de ensino, aproximadamente 60 alunos de quatro turmas de graduação criaram aplicações para a mesa tangível com o Eduba Editor para diferentes tipos de deficiências: visual, auditiva, intelectual e autismo. Nas duas escolas públicas, 16 professores participaram da formação para uso dessa tecnologia e as aplicações desenvolvidas por eles e pela nossa equipe foram aplicadas para 12 alunos com deficiência intelectual ou autismo, estudantes da educação básica e da EJA. A aceitação e o uso deste sistema de interação tangível pelos professores ainda estão sendo avaliados com os questionários TAM (Technology Acceptance Model) e TPACK (Technological Pedagogical Content Knowledge).

\section{Uso de Mesa Tangível de Baixo Custo na Educação Inclusiva}

Uma mesa tangível pode ser utilizada com resultados positivos na educação inclusiva se forem observados três requisitos:

a) montar uma mesa tangível com custos viáveis;

b) utilizar uma solução de software que permita que o próprio professor desenvolva seus recursos educacionais para a mesa tangível;

c) proporcionar uma formação dos professores para o uso de interações tangíveis no contexto inclusivo que faça com que os mesmos sejam capacitados e sintamse familiarizados e motivados a usar essa tecnologia.

\subsection{Montagem de uma mesa tangível de baixo custo}

Uma mesa tangível de baixo custo (fig. 2) pode ser desenvolvida com os seguintes componentes e materiais:

- Projetor: (p) 1 mini projetor (1600 lúmens ou mais) ou projetor de curta distância (2500 lúmens ou mais). O projetor deve proporcionar uma projeção maior que $36 "(80 \mathrm{~cm} \times 50 \mathrm{~cm})$ a $120 \mathrm{~cm}$ de distância e permitir fixação em sua base com 2 ou 3 parafusos.

- Câmera IR: (c) 1 câmera infravermelho com conexão USB e lente $28 \mathrm{~mm}$ com suporte m12. Uma webcam convencional normalmente tem um filtro que impede a entrada de IR no sensor e deve ser evitado seu uso, mas pode ser utilizada, desde que esse filtro, localizado entre a lente e o sensor seja removido.

- Filtro IR: (f) 1 filtro passa-alta IR $850 \mathrm{~nm} 12 \mathrm{~mm}$, colocado entre a lente e o sensor da câmera, para permitir somente a passagem de IR impedindo a entrada da luz convencional no sensor da câmera. Um filme de fotografia velado ou a parte bem escura de uma radiografia pode ser usado como filtro IR.

- Iluminador Infravermelho: (i) 2 peças com fonte de alimentação $12 \mathrm{~V}$, colocados de modo a iluminar para baixo, para que a iluminação seja difusa.

- Acrílico transparente: (a) 1 peça $80 \mathrm{~cm}$ x $60 \mathrm{~cm} \times 2 \mathrm{~mm}$.

- Vinil translúcido: (v) 1 peça $85 \mathrm{~cm}$ x $65 \mathrm{~cm}$. Pode ser usado vinil translúcido, película para retroprojeção em vitrines ou, alternativamente, papel vegetal coberto com vinil ou plástico PVC transparente (cristal) simples. 
- MDF 15mm: (m1) 2 peças $80 \mathrm{~cm} \times 74 \mathrm{~cm}$, com 4 furos na peça traseira para ventilação; (m2) 2 peças $57 \mathrm{~cm}$ x $74 \mathrm{~cm}$; (m3) 2 peças $25 \mathrm{~cm}$ x $57 \mathrm{~cm} ;(\mathrm{m} 4) 1$ peça $42 \mathrm{~cm} \times 47 \mathrm{~cm}$; (m5) 1 peça $40 \mathrm{~cm} \times 30 \mathrm{~cm}$.

- MDF 6mm: (mp) 1 peça $78 \mathrm{~cm}$ x $58 \mathrm{~cm}$, para a proteção da superfície da mesa, quando ela não estiver em uso.

- Espelho: (e) 1 peça $2 \mathrm{~mm}$ x $40 \mathrm{~cm}$ x $30 \mathrm{~cm}$, colado no MDF com fita dupla-face.

- Cantoneiras L para móveis $35 \mathrm{~mm}$ ou similar: (L) 16 peças.

- Dobradiças para móveis $60 \mathrm{~mm}$ ou similar: (d) 4 peças.

- Parafusos para madeira 12mm: (pm) 96 peças, para fixar as cantoneiras e dobradiças.

- Parafusos $80 \mathrm{~mm}$ com arruela e porca: (pr) 4 parafusos, 8 arruelas, 8 porcas, para a regulagem do ângulo do projetor e do espelho.

- Moldura de madeira $6 \mathrm{~cm}$ x $3 \mathrm{~cm}:(\mathrm{mm}) 2$ peças $80 \mathrm{~cm} ; 2$ peças $60 \mathrm{~cm}$

- Sapata ou ponteira em formato de U (pé) para MDF de $15 \mathrm{~mm}$ : (u) 4 peças.

- Computador ou notebook: (n) 1 equipamento para instalação do software e conexão com projetor e câmera.

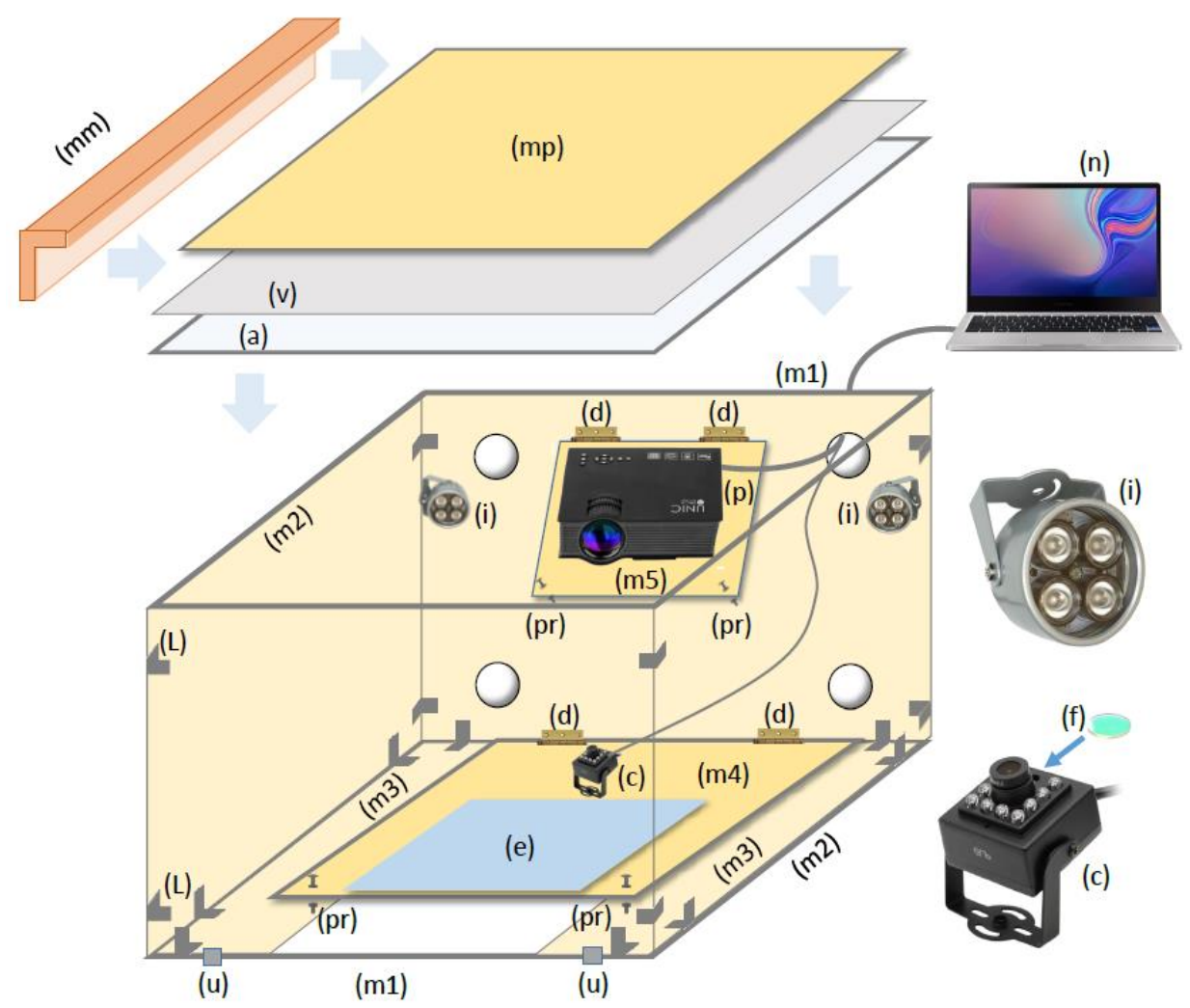

Figura 2. Especificação para montagem de uma mesa tangível de baixo custo

\subsection{Utilização de um editor de recursos educacionais tangíveis}

Para permitir a criação dos recursos educacionais tangíveis de modo interativo e intuitivo e a sua execução numa mesa tangível, foi desenvolvido o Eduba Editor (PREUSS et al., 2019). O software do editor e do player, a documentação e os tutoriais de uso do mesmo estão disponíveis no site do projeto (Eduba Editor, 2020). 
No Eduba Editor, as aplicações são formadas por um conjunto de cenas. Em cada cena são definidas e configuradas as imagens de fundo, os áudios de narração ou explicação e as demais imagens ou vídeos que compõem o cenário. Quando há interação com objetos tangíveis, são delimitadas no editor as áreas para a interação e a configuração dos elementos de marcação fiducial corretos e incorretos e o respectivo feedback sonoro ou pictográfico para cada resposta. De acordo com a interação, além do feedback, a próxima cena ou uma cena específica pode ser exibida.

O editor (fig. 3-a) possui uma interface para o desenvolvimento das aplicações na qual os elementos de texto, imagens, vídeos e áreas de interação são inseridos de forma interativa com o uso do mouse, clicando, arrastando, dimensionando e preenchendo as propriedades, de acordo com o contexto de cada elemento. Ao final da edição, uma aplicação no formato HTML5 é gerada para ser executada na mesa tangível, projetando a imagem na superfície da mesa e reagindo à colocação e movimentação dos objetos com marcadores fiduciais (fig. 3-b) sobre a mesa.

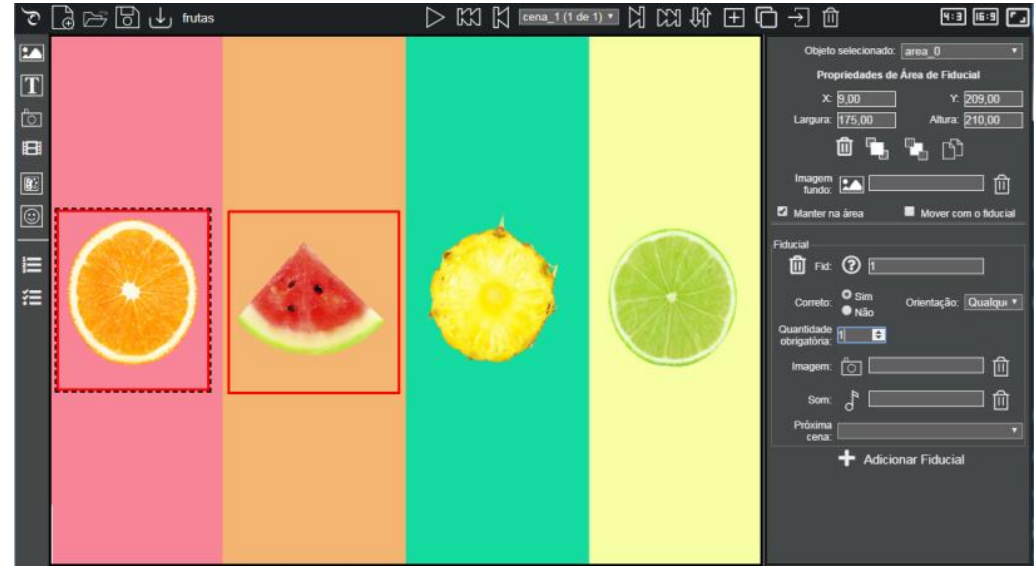

(a)

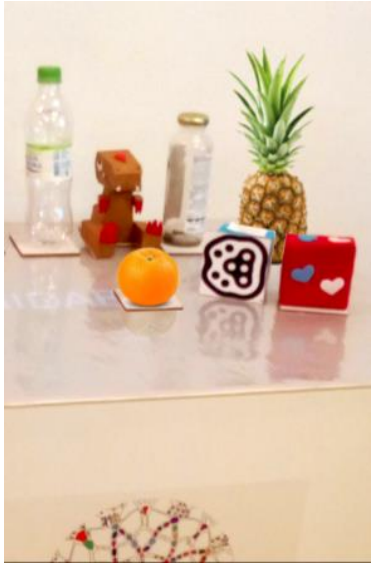

(b)

Figura 3. Interface do editor e objetos com marcadores fiduciais

\subsection{Formação dos professores para uso de recursos tangíveis}

Para um uso eficiente dos recursos tangíveis na educação inclusiva é necessário colocar em prática um modelo de formação de docentes que propicie elementos teóricos e metodológicos para apoiar a integração propositiva crítica dos recursos tecnológicos digitais à prática pedagógica. Nesse sentido, os programas e as políticas que instigam esse processo precisam também contemplar os percursos formativos e organizacionais que envolvem o uso de tecnologias em sala de aula para facilitar e ampliar a efetiva inclusão de alunos com deficiência.

Para a criação de uma aplicação para a mesa tangível usando o editor é necessário um planejamento, no qual estejam bem definidos os objetivos propostos e a quantidade de cenas que ela terá. Para cada cena, devem ser definidos: o objetivo, as imagens, vídeos, textos e áudios que irão instruir e orientar o aluno sobre a atividade, além da especificação das áreas de interação e seus respectivos feedbacks.

O que difere uma mesa tangível de um tablet é a possibilidade de uso de objetos reais para a interação. Assim, esses objetos devem ser significativos e coerentes com a interação e objetivos pretendidos, o que requer atenção e estudo. É importante a 
realização de uma capacitação de professores para atividades tecnológicas específicas, como o tratamento de imagens digitais (recortar, inverter, deixar fundo transparente, criar GIFs animados), edição de áudio (gravar, cortar, reduzir o ruído, normalizar) e edição de vídeo (editar, cortar, retirar ou separar o áudio, legendar), pois esses elementos são necessários para o desenvolvimento das aplicações para a mesa tangível.

\subsection{Resultados e Discussões}

Esta proposta apresenta uma solução viável de construção e utilização de uma mesa tangível resistente, que pode ser desmontada e transportada com facilidade. A moldura no tampo, além de fixar o acrílico e evitar que ele se desloque ou caia, serve de guia para a colocação de uma proteção na superfície, para quando a mesma não estiver em uso. $\mathrm{O}$ uso de câmera com suporte para lente $\mathrm{m} 12$ facilita a colocação do filtro IR por ser rosqueável. Os iluminadores IR fornecem uma iluminação difusa e o seu posicionamento evita que seu reflexo no espelho e no tampo seja captado pela câmera. Este modelo de mesa apresenta custos viáveis, visto que as nossas duas mesas que utilizaram mini projetores de 1600 lumens tiveram custos abaixo de $\mathrm{R} \$ 2.000,00$ cada, não incluindo o custo do notebook ou computador. A mesa que utilizou um projetor de 3000 lúmens apresentou um custo um pouco superior a $\mathrm{R} \$ 3.000,00$.

A proposta também inclui um software intuitivo para que o próprio professor possa criar os recursos de acordo com as necessidades educacionais de seus alunos. $\mathrm{O}$ editor foi criado com várias funcionalidades a mais que o KitVision, principalmente uma interface mais intuitiva, suporte a diversos formatos de imagem, áudio e vídeo, facilidade para criar animações, mais flexibilidade para criar aplicações não lineares, mais opções de feedback, um simulador para testar as aplicações, uma versão off-line e uma versão web que permite o uso do editor também em dispositivos móveis e em diferentes sistemas operacionais.

Toda a documentação e tutoriais para instalação, configuração do software e produção de recursos educacionais tangíveis estão disponíveis no site do projeto (Eduba Editor, 2020). O material para a formação de professores, baseado em textos, exemplos e tutoriais, está em fase final de desenvolvimento e a formação será contínua e disponível no MOOC da instituição.

Os resultados parciais indicam a viabilidade e utilidade de interação tangível na educação inclusiva. Os resultados da avaliação de usabilidade (System Usability Scale SUS) do editor são bons (acima de 70 pontos), o que indica que ele está pronto para ser utilizado. Os resultados da avaliação da motivação e autonomia (Intrinsic Motivation Inventory - IMI) na criação de aplicações para mesa tangível indicam a importância desse tipo de atividade na educação inclusiva e ressaltam a necessidade da formação de professores para uso desta tecnologia.

\section{Conclusão}

As mesas de interação tangível podem ser utilizadas na educação inclusiva como um recurso de tecnologia assistiva se houver uma mesa tangível com um custo viável, um software adequado e uma formação que capacite e motive os professores a utilizarem essa tecnologia. Isso pode auxiliar no processo de ensino e aprendizagem de alunos com ou sem deficiência, proporcionando atividades mais enriquecedoras e interativas. 
Foi apresentada uma proposta de utilização da mesa tangível na educação inclusiva, contemplando uma especificação para montagem de uma mesa de baixo custo e o uso de um editor interativo e intuitivo especificamente desenvolvido para esse fim. Além disso, foram abordados os aspectos relacionados à formação dos professores e sua importância para o uso eficiente no planejamento, criação e utilização deste tipo de tecnologia.

Os próximos passos desta pesquisa e do desenvolvimento do software envolvem a criação de um repositório para compartilhamento das aplicações desenvolvidas e a finalização das avaliações e análise dos dados sobre seu uso e aceitação da tecnologia.

\section{Agradecimentos}

O presente trabalho foi realizado com apoio do Ministério da Ciência, Tecnologia e Inovações, da Coordenação de Aperfeiçoamento de Pessoal de Nível Superior - Brasil (CAPES) - Código de Financiamento 001, do Conselho Nacional de Desenvolvimento Científico e Tecnológico (CNPq) e da Fundação de Amparo à Pesquisa do Estado do Rio Grande do Sul (FAPERGS).

\section{Referências}

Baldassarri, S.; Cerezo, E.; Beltrán, J. R. (2017) "Immertable: A Configurable and Customizable Tangible Tabletop for Audiovisual and Musical Control", In: Proceedings of the XVIII International Conference on Human Computer Interaction (Interacción '17). ACM, New York, USA, Article 32, p. 1-8.

Baldassarri, S. et al. (2018) "Videogame-based Case Studies for Improving Communication and Attention in Children with ASD", In: Proceedings of the XIX International Conference on Human Computer Interaction (Interacción 2018). ACM, New York, USA, Article 9, p. 1-8.

Bonillo, C. et al. (2016) "Tangible Activities for Children with Developmental Disorders", In: Proceedings of the XVII International Conference on Human Computer Interaction (Interacción '16). ACM, New York, USA, Article 9, p. 1-2.

Bonillo, C. et al. (2019) "KitVision toolkit: supporting the creation of cognitive activities for tangible tabletop devices", Universal Access in the Information Society v. 19, p. 361-389.

Cerezo, E. et al. (2019) "Guidelines to design tangible tabletop activities for children with attention deficit hyperactivity disorder", International Journal of Human Computer Studies, v. 126, p. 26-43.

Chen, J. et al. (2019) "A pilot study on evaluating children with autism spectrum disorder using computer games", Computers in Human Behavior, v. 90, p. 204-214.

Eduba Editor (2020) "Eduba Editor". Disponível em: https://nidaba.online. Acesso em: 30 jun. 2020.

Falcão, T. P. (2018) "Feedback and Guidance to Support Children with Intellectual Disabilities in Discovery Learning with a Tangible Interactive Tabletop", ACM Trans. Access. Comput., v. 11, n. 3, p. 16:1-16:28. 
Gluz, J. et al. (2018) “Ambiente Virtual Tangível para Integração Sensorial no Ensino de Ciências numa Perspectiva Inclusiva", Simpósio Brasileiro de Informática na Educação - SBIE, v. 29, n. 1, p. 545. DOI: 10.5753/cbie.sbie.2018.545.

Jordà, S. et al. (2006) "The reacTable: A Tangible Tabletop Musical Instrument and Collaborative Workbench". In ACM SIGGRAPH 2006 Sketches (SIGGRAPH '06). ACM, New York, USA, p. 91-es.

Jung, J. et al. (2013) "E-CORE (Embodied COgnitive REhabilitation): A cognitive rehabilitation system using tangible tabletop interface". In: Pons J., Torricelli D., Pajaro M. (eds) Converging Clinical and Engineering Research on Neurorehabilitation. Biosystems \& Biorobotics, v. 1. Springer, Berlin, Heidelberg.

Kaltenbrunner, M. (2009) "reacTIVision and TUIO: A Tangible Tabletop Toolkit". 2009. In Proceedings of the ACM International Conference on Interactive Tabletops and Surfaces (ITS '09). ACM, New York, USA, p. 9-16.

Korozi, M. et al. (2018), "Designing an augmented tabletop game for children with cognitive disabilities: The "Home game" case". British Journal of Educational Technology, 49, p. 701-716.

Marco, J.; Baldassarri, S.; Cerezo, E. (2013) "NIKVision: Developing a Tangible Application for and with Children”, J. UCS, v. 19, n. 15, p. 2266-2291.

Marco, J.; Cerezo, E.; Baldassarri, S. (2016) "Lowering the threshold and raising the ceiling of tangible expressiveness in hybrid board-games", Multimedia Tools and Applications, v. 75, n. 1, p. 425-463.

Preuss, E. et al. (2019) "E-DUB-A: A Tangible Educational Resource Editor in Inclusive Classes". In: 2019 IEEE 19th International Conference on Advanced Learning Technologies (ICALT), Maceió, Brazil, 2019, p. 303-307. DOI: 10.1109/ICALT.2019.00095.

Samsung Electronics (2011) "Samsung SUR40 for Microsoft Surface: User Manual". Disponível em: http://downloadcenter.samsung.com/content/UM/201206/20120622 172918410/BN46-00030A-Eng.pdf. Acesso em: 26 jun. 2020.

Schneider, B.; Blikstein, P.; Mackay, W. (2012) "Combinatorix: A Tangible User Interface That Supports Collaborative Learning of Probabilities". In Proceedings of the 2012 ACM international conference on Interactive tabletops and surfaces (ITS '12). ACM, New York, USA, p. 129-132.

Schöning, J. et al. (2010) "Building Interactive Multi-touch Surfaces". In: MüllerTomfelde, C. (Org.), Tabletops - Horizontal Interactive Displays, London, Springer London. p. 27-49.

Shaer, O.; Hornecker, E. (2009) "Tangible User Interfaces: Past, present, and future directions", Foundations and Trends in HCI, v. 3, n. 1-2, p. 1-137.

Ullmer, B.; Ishii, H. (2000) "Emerging frameworks for tangible user interfaces", IBM Systems Journal, v. 39, n. 3-4, p. 915-930.

Vygotsky, L. S. Obras Escogidas V: Fundamentos de defectología. $1^{\mathrm{a}}$ ed. Madrid, Antonio Machado, 2012. 